\title{
ROTATION-PLASTY FOR CONGENITAL DEFEGTS OF THE FEMUR
}

\section{Making use of the Ankle of the Shortened Limb to Control the Knee Joint of a Prosthesis}

\author{
C. P. Van Nes, Leidex, Holland \\ From the Orthopaedic Department, Eniversity of Leiden
}

Several varieties of hypoplasia or congenital absence of part of the femur are seen in orthopaedic practice. There may be delayed development and shortening of the whole femur with subluxation of the hip or knee joint (Fig. I) but sometimes with these joints well developed. In other cases the lower end of the femur may be present but the upper end is luxated and not differentiated into a head and neck (Fig. 2). Finally the middle part of the femur may be absent, the short proximal fragment being abducted and the knee joint

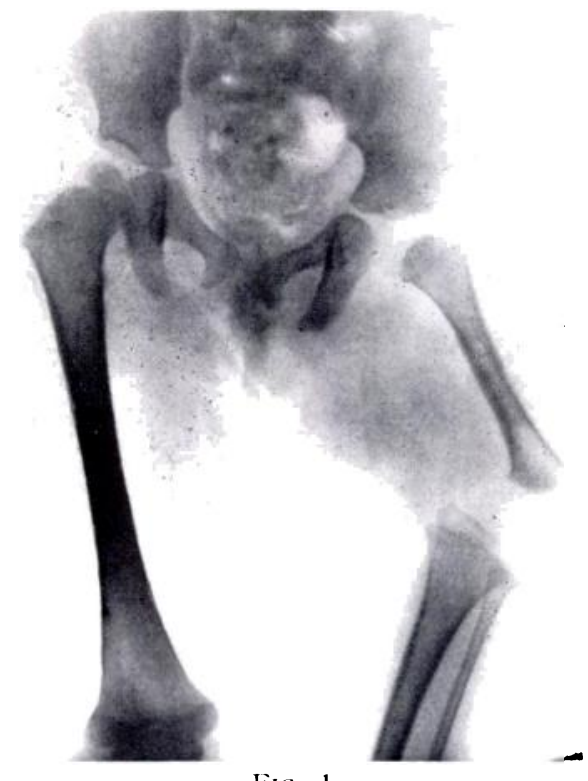

FIG. 1

Delaved development and shortening of the femur with congenital subluxation of the knee joint.

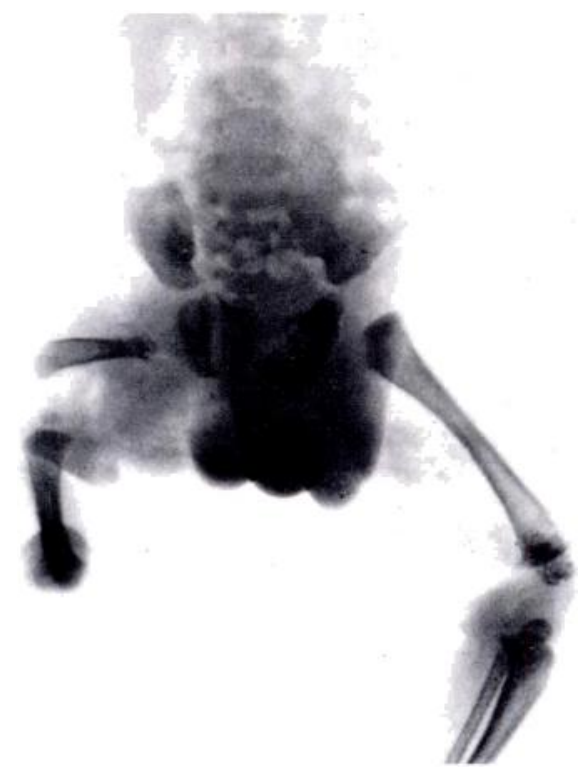

Fig. 2

Failure of development of the upper end of the femur with shortening of the bone and congenital subluxation of the hip joint.

situated just below the hip (Fig. 3). In every type of congenital defect of the femur there is severe shortening of the thigh so that the ankle joint of the short limb is at about the level of the knee joint of the normal side.

To enable these patients to walk we must provide them with an appliance by which to compensate for the shortening. But since the knee joint of the defective limb is situated immediately beneath the hip, and is related to a large mass of muscle, it is seldom possible to allow free mobility of the knee in any prosthesis. Furthermore, since the ankle joint, which is now at the level of the sound knee, permits only a useless range of hyperextension movement, the ankle must also be immobilised. There is no alternative but to fit a leather cuff to the disabled limb with the foot in equinus and to extend the appliance to the necessary length by means of a stilt or other device. It is difficult to fit the leather cuff to the thigh, which is short and thick, so that a pelvic band is often necessary. Thus, finally, the prosthesis 


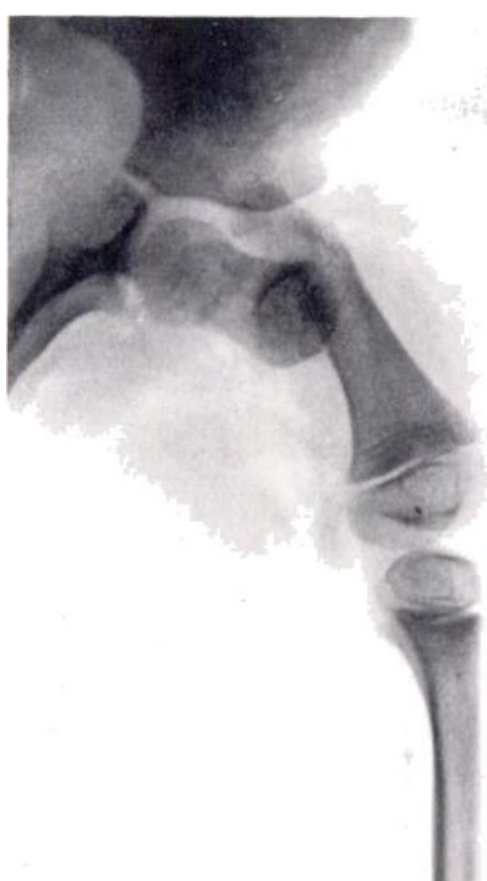

FII; 3

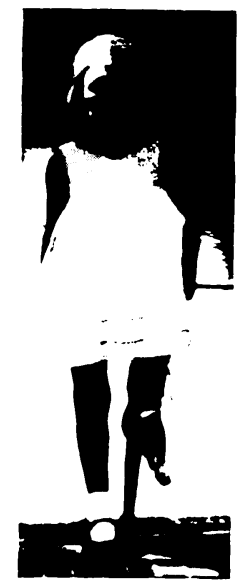

Fici. 4

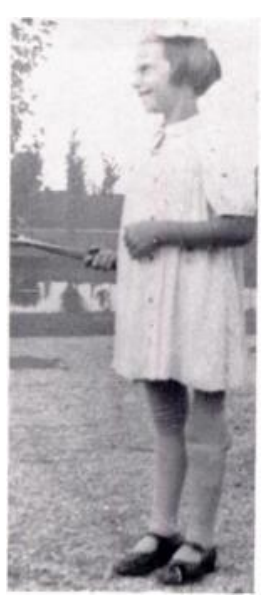

III, 6

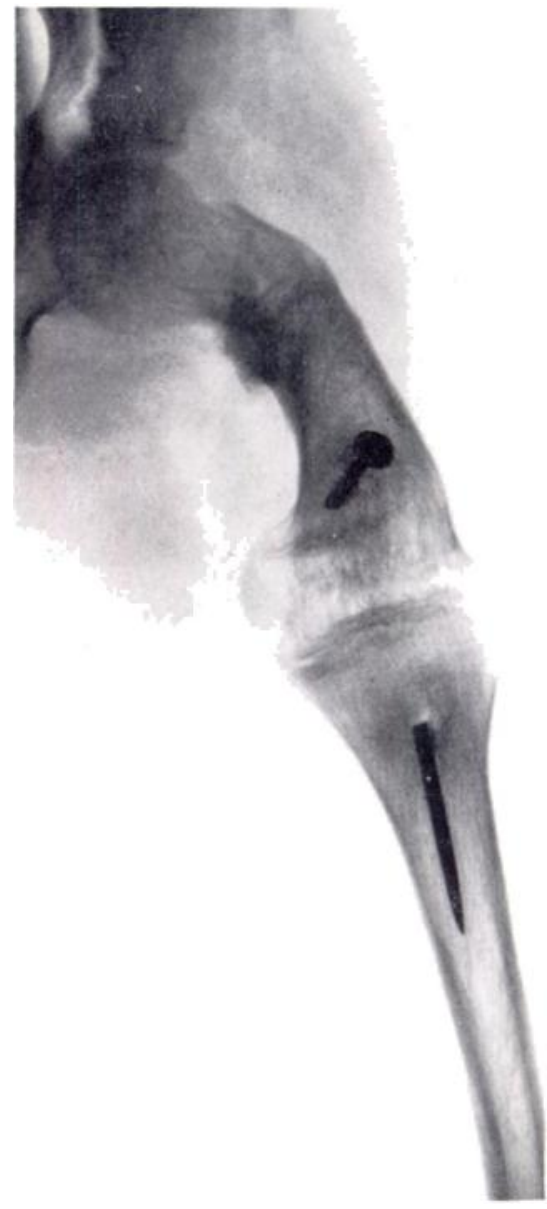

Fici. 5 Case 1. (ongenital absence of the midelle
part of the femur (Fig. 3 ). The child
learned to walk on a pron (Fig. 4). Ifter
fusion of the pseudarthrosis and rotation-
plasty, and later tibio-femoral fusion
(Fig. 5), she was titted with a prosthesis
with the ankle joint of the shortened limb at the level of the knee (Fin. 6).

is rigid and heary, and it permits only flexion and extension movement of the hip joint. Walking is difficult and tiresome, particularly since the hip muscles are often imperfectly developed, and the prosthesis itself is unsightly and repellent especially when there is fixed abduction-flexion deformity at the hip. Every patient who is fitted with such an apparatus complains of the difficulties and inconvenience of attempting to travel in public converances. We have therefore tried to find other ways of improving the lot of these disabled persons.

The shortening is of such a degree that it cannot be compensiated by lengthening the short femur or shortening the long femur. Borggreve (1930) suggested a method of overoming the main difficulty. In a patient with thirty-five centimetres of shortening of the lower limb due $t o$ resection of bone at an early age, he rotated the limb through 180 degrees, so that the heel was directed forwards and the toes backwards. The muscles controlling the ankle of the short limb could then work the knee joint of the prosthesis.

VOL. $32 \mathrm{~B}$, NO. 1, FEBRLARY 1950 
The difficulties I met in applying this idea to cases of shortening from congenital defects of the femur were even greater than those reported by Borggreve. He had only to rotate the leg through 180 degrees, whereas most of my patients also required treatment of the defect in the femur. In the first place it was sometimes necessary to secure union of a pseudarthrosis of the femur. Secondly, it was often impossible to stabilise the knee in its position immediately under the hip joint, in the bulky tissues of the thigh, so that fusion of the knee was necessary. Much of the required rotation of the limb could be gained in these two operations, but if $\mathbf{1 8 0}$ degrees of rotation had not been attained it was necessary finally to perform a rotation osteotomy so that the heel was directed forwards. A combination of these operations has been practised several times at the Anna Kliniek with very satisfactory results. I will describe in detail three patients whose photographic records are complete. All were treated many years ago and the results can now be considered as final.

\section{CASE REPORTS}

Case 1. Female, aged 1 year-First seen in 1929, at the age of one year, with considerable shortening of the lower limb from a defect of the shaft of the femur (Fig. 3). The left femur measured 8 centimetres, the right 24 centimetres. The thigh was abducted and flexed 45 degrees. With a walking appliance consisting of a simple leather pylon the patient learned to walk with the hip and knee in slight flexion (Fig. 4). In succeeding years the appliance became increasingly troublesome and it was decided to perform the operation that we have described as " rotation-plasty."

In June 1935 the pseudarthrosis of the femur was exposed. The fragments were freshened and osteosynthesis was carried out. At the same time the lower leg was rotated so that the heel faced forwards. Some months later the patient was given a prosthesis with a knee joint which she moved with the flexors and extensors of the foot (Fig. 6). It was impossible to control the high knee joint and bulky thigh muscles so that arthrodesis of this joint was performed in January 1937 (Fig. 5). The child walked very well with her new apparatus and was able to participate in sports. In the winter of 1940, with much confidence but some imprudence, she engaged in skating and fell, sustaining a fracture of the short femur. This was treated in another hospital, with the result that some rotation was lost. Nevertheless she can still walk several miles, is skilled in housekeeping, is on her feet all day, and intends soon to marry.

Case 2. Female, aged 9 years-This patient attended our out-patient department in 1928, at the age of nine years, with severe shortening of the left lower limb due to a congenital defect of the femur. There was pseudarthrosis of the femoral shaft and the thigh was abducted nearly 90 degrees (Fig. 7). She was supplied with a walking appliance including a pelvic belt. To give good support during walking it was necessary to immobilise all three articulations of the short limb (Fig. 8).

In 1937 she complained of pain in the pseudarthrosis and we decided to perform a rotation-plasty. The first operation was done in May 1937. To correct the flexion-abduction deformity of the thigh, and to raise the foot to the level of the sound knee, the distal part of the femur was excised. The proximal fragment of the femur and the upper part of the tibia were freshened and after rotating the limb through 90 degrees the bones were fixed by two screws. Eight days later we performed a rotation osteotomy of the tibia which brought the heel into the forward position. Some months later the patient was fitted with a prosthesis with a free knee articulation. (Fig. 9). She soon walked very well, moving her new " knee joint " physiologically. A slight limp remained as a result of the weakened hip muscles.

Case 3. Female, aged 15 years-First seen in 1936 with twenty-eight centimetres of shortening of the right lower limb from hypoplasia of the femur (Fig. 10). The ankle was four centimetres below the level of the sound knee joint. She then wore a heavy and rigid prosthesis which enveloped the whole limb (Fig. 11). Walking was difficult and the patient was anxious to have something done. We proposed rotation-plasty and this was performed in September 1939. After resecting four centimetres of bone at the knee, the limb was rotated through 180 degrees and fixed by a Steinmann's pin. Some months later the patient was fitted with a prosthesis with a free knee articulation (Fig. 12). She became accustomed to this apparatus very quickly and now walks normally.

These three patients, all of whom were treated eight to ten years ago, report regularly to our clinic for repair or renewal of their prostheses. We have therefore had the opportunity of reviewing them from time to time, and the late-results have remained excellent. The patients are very satisfied and grateful for the aesthetic, functional and social improvements they have gained. Other cases, operated upon in a similar way in more recent years, have also secured gratifying results but are not presented in detail because, in consequence of war, 


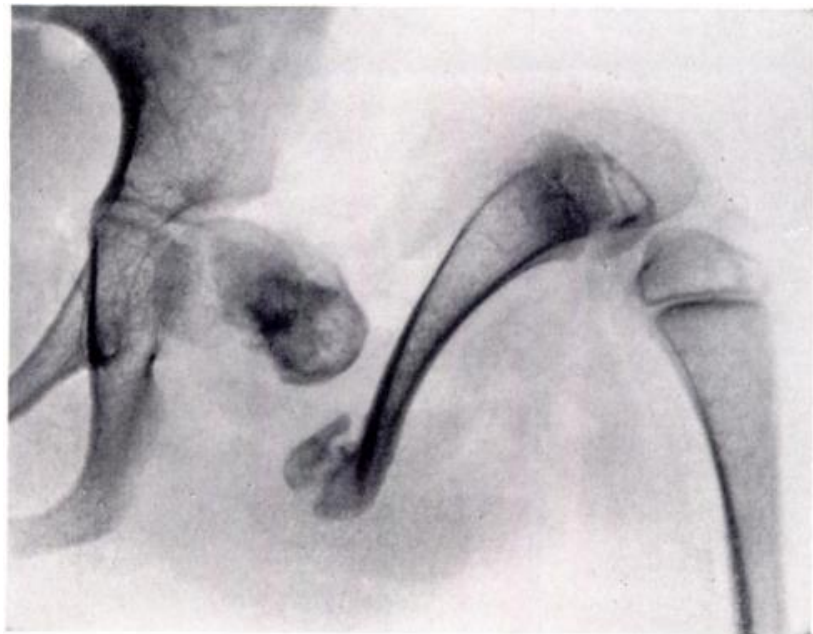

FIG. 7

Case 2. Congenital hypoplasia of the femur with pseuclarthrosis in the miclelle third.

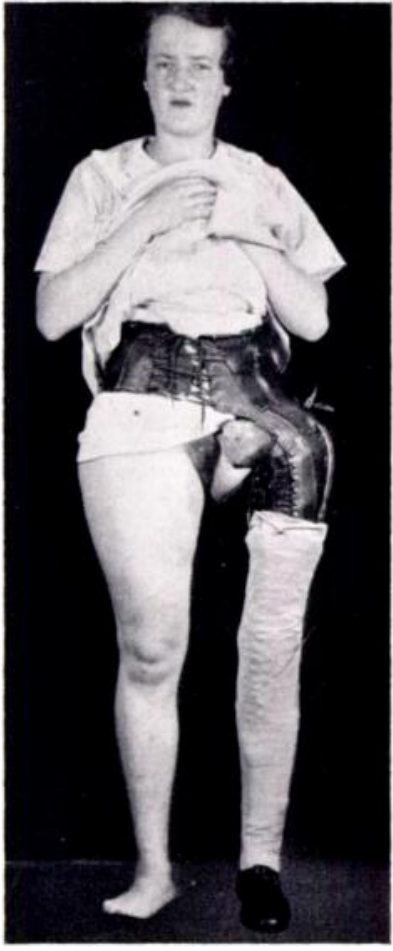

FIG. \&

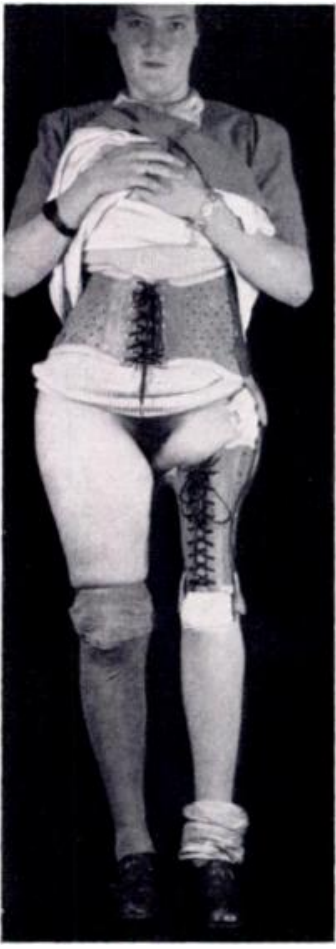

FIG. 9

(ase 2. The walking appliance that was originally fitted to the abducted stump immobilised the hip, knee and ankle joints (Fig. 8). After excision of the distal fragment of the femur. fusion of the proximal fragment to the tibia, and rotation-plasty, an artificial limb was fitted with good control at the level of the knee by the rotated ankle joint of the shortened limb (Fig. 9). 


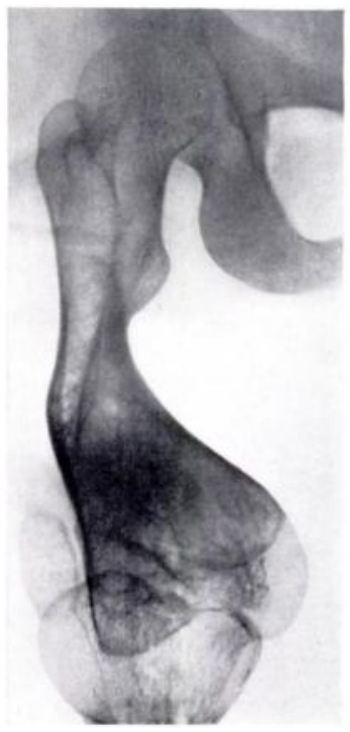

Fis. 10

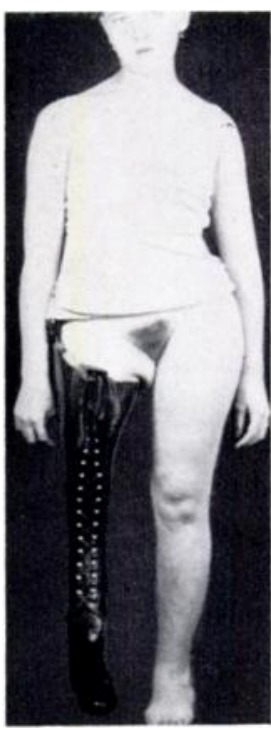

Fici. 11

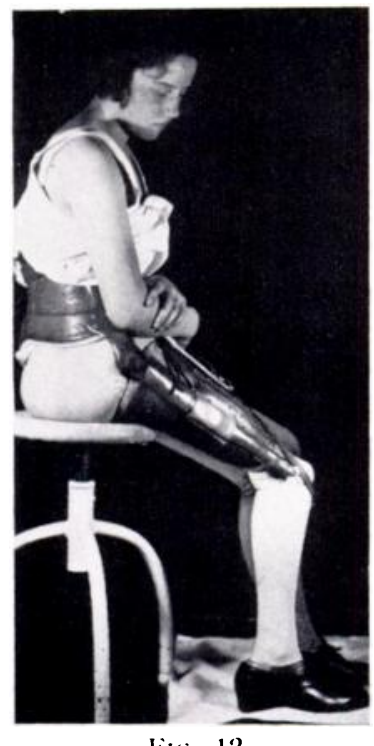

Fici. 12

Case 3. Congenital hypoplasia of the femur, the ankle joint being 4 centimetres below the level of the sound knee joint (Fig. 11). I heav and rigid prosthesis had been fitted (Fig. 11). Ifter fusion of the knee with resection of 4 centimetres of bone, and "rotation-plasty" "through 180 degrees, a jointed prosthesis was fitted, the ankle joint of the short limb controlling the knee of the prosthesis (Fig. 12).

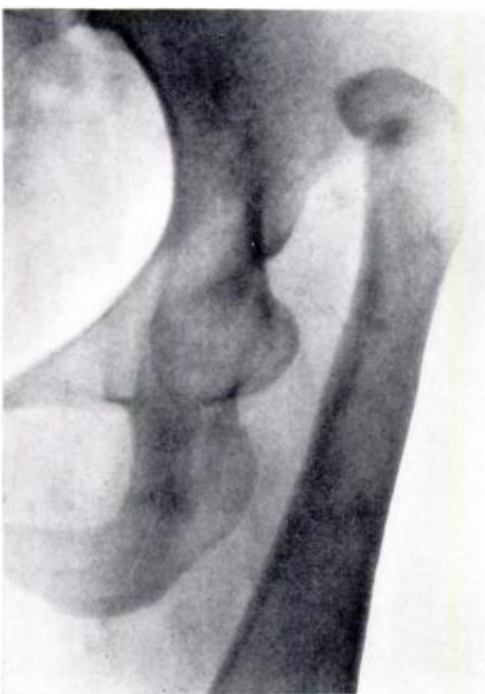

FIs. 13

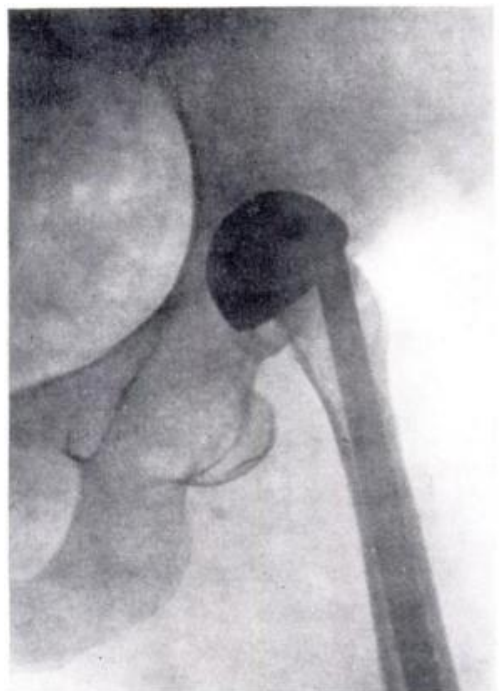

Fig. 14

Congenital hrpoplasia with vers marked shortening of the femur and dislocation of the hip joint (Fig. 13). " Rotation-plasty" "with intramedullary nail fixation was combined with reposition of the hip joint with a vitallium cup (Fig. 14).

many of their clinical records and photographs have been destroved. Intramedullary pin fixation is now used in most cases, thus giving the possibility of sound internal fixation with rotation of the limb at the same time. One such case is shown in Figures 13 and 14 . There was hypoplasia of the femur with congenital dislocation of the hip joint. "Rotation-plasty" was performed with intramedullary pin fixation and reposition of the hip with vitallium cup arthroplasty.

Borgigrae, J. (1930): Archiv für orthopädische Chirurgie, 28, 175. 